Revista Brasileira de

Engenharia Agrícola e Ambiental

v. 15, n.1, p.53-59, 2011

Campina Grande, PB, UAEA/UFCG - http://www.agriambi.com.br

agriambi Protocolo 197.09 - 21/12/2009 • Aprovado em 22/10/2010

\title{
Efeito do estresse salino em genótipos tetraplóides de bananeira
}

\author{
Lilia W illadino', Eline W. F. Gomes ${ }^{2}$, Énio F. de F. e Silva ${ }^{3}$, \\ Luiza S. S. Martins ${ }^{1} \&$ Terezinha R. Camara ${ }^{4}$
}

\begin{abstract}
RESU MO
0 presente trabalho avaliou 12 genótipos de bananeira no que se refere à tolerância à salinidade em fase inicial de crescimento, período do ciclo fenológico de maior sensibilidade. As plantas foram cultivadas, durante 21 dias, e submetidas a um tratamento com $100 \mathrm{mM}$ de $\mathrm{NaCl}$ e a um controle, sem $\mathrm{NaCl}$. Avaliaram-se atributos biométricos e teores de elementos minerais no tecido vegetal. Seis genótipos (Calipso, FHIA-02, PA 42-44, Bucaneiro, FHIA-03 e PV 42-142) dos doze estudados, apresentaram sintomas de toxidez causados pelo tratamento salino. O genótipo PA 42-44 se destacou como mais sensível, com os maiores teores de $\mathrm{Na}^{+}$, tanto no limbo foliar quanto nas raízes e rizoma, associados a uma redução da massa seca, da ordem de 18,5\%. O s elevados teores de sódio detectados no genótipo PA 42-44 sugerem uma eficiência menor na extrusão do cátion paralelamente à ausência de habilidade em evitar sua translocação para o limbo foliar. Por outro lado, o genótipo Preciosa apresentou os menores teores de $\mathrm{Na}^{+}$e a menor redução da produção de biomassa seca $(0,2 \%)$, além de baixa relação $\mathrm{Na}^{+} / \mathrm{K}^{+}$.
\end{abstract}

Palavras-chave: Musa spp, potássio, sódio, relação $\mathrm{Na}^{+} / \mathrm{K}^{+}$

\section{Effect of salt stress on banana tetraploid genotypes}

\begin{abstract}
This study evaluated 12 banana genotypes with respect to salt tolerance during initial growth, the most sensitive stage of the phenological cycle. Plants were grown for 21 days under $100 \mathrm{mM} \mathrm{NaCl}$ or under a control treatment without $\mathrm{N} \mathrm{aCl}$. Biometrical parameters and mineral contents in plant tissue w ere evaluated. Six genotypes (Calipso, FH IA-02, PA 42-44, Bucaneiro, FHIA-03 and PV 42-142) out of 12 considered in this study showed toxicity symptoms induced by saline treatment. The PA $42-44$ genotype was pointed out as the most sensitive one because it showed the highest $\mathrm{Na}^{+}$contents in both leaf blade and roots and rizome, besides a $18.5 \%$ reduction of dry matter production. The high $\mathrm{N} \mathrm{a}^{+}$contents found for PA $42-44$ tissues suggest a low efficacy to extrude and to prevent the $\mathrm{Na}^{+}$translocation to leaf blade. On the other hand, the Preciosa genotype showed both the lowest $\mathrm{Na}^{+}$contents and the smallest reduction for dry matter production $(0.2 \%)$ as well as a low $\mathrm{Na}^{+} / \mathrm{K}^{+}$ratio indicating a salt tolerance strategy by $\mathrm{Na}^{+}$ extrusion.
\end{abstract}

Key words: Musa spp, potassium, sodium, $\mathrm{Na}^{+} / \mathrm{K}^{+}$ratio

\footnotetext{
DB/UFRPE Rua D. Manoel de Medeiros s/n, Dois Irmãos. CEP 52171-900 Recife, PE, e Instituto Nacional de Ciência e Tecnologia em Salinidade (INCTSal/CN Pq) Fone: (81) 3320 6366. E-mail: lilia@pesquisador.cnpq.br, luizasemen@ hotmail.com

2 Empresa Pernambucana de Pesquisa Agropecuária, Av. General San Martin, n. 1371, Bonji. CEP 50671-000 Recife, PE. E-mail: elinewaked@hotmail.com

${ }^{3}$ DTR/U FRPE e IN CTSal/CN Pq. E-mail: enio.silva@pq.cnpq.br

${ }^{4}$ DQ/UFRPE. E-mail: tkrcamara@bol.com.br
} 


\section{INTRODUÇÃO}

A bananicultura é uma atividade desenvolvida, atualmente, em mais de 100 países tropicais e subtropicais, e apresenta uma produção mundial de aproximadamente 80 milhões de toneladas de fruta fresca, em área colhida de 4 milhões de hectares (UNCTAD, 2009). No Brasil a cultura da banana ocupa o segundo lugar em volume de frutas produzidas e a terceira posição em área colhida. A cultura da bananeira, todavia, apresenta significativa limitação de produção quando cultivada em solos salinizados. Atualmente, cerca de 900 milhões de hectares de terras do planeta são afetados por sais (Flowers, 2004). No Brasil, na década de oitenta cerca de 160 mil hectares já haviam sido registrados como salinizados, dos quais 89.000 se situam na região Nordeste. Este panorama evidencia a necessidade de se incrementar os estudos sobre as estratégias de tolerância de plantas ao estresse salino. A capacidade de adaptação é fundamental, envolve mudanças fisiológicas, morfológicas e enzimáticas da planta e organelas. Essas respostas metabólicas requerem horas, dias ou semanas (Munns et al., 2002).

A salinização dos solos resulta na redução do acúmulo de fitomassa, o que reflete, em parte, o custo metabólico de energia associado às adaptações da condição de estresse salino, incluindo: a síntese de solutos orgânicos para osmorregulação e/ou proteção de macromoléculas, a manutenção da integridade das membranas e a regulação do transporte e distribuição iônica em vários órgãos e dentro das células (Willadino \& Camara, 2004). O controle da distribuição do $\mathrm{Na}^{+}$nos órgãos e tecidos da planta, bem como no interior da célula, se dá em função da atividade dos transportadores $\mathrm{de} \mathrm{Na}^{+} / \mathrm{H}^{+}$que, dependendo do nível de salinização, garantem a manutenção da homeostase iônica (Shi et al., 2002). A exclusão do $\mathrm{Na}^{+}$através da membrana plasmática resulta na extrusão do $\mathrm{Na}^{+}$para outras células ou tecidos ou para a solução do solo. O efeito da extrusão do $\mathrm{Na}^{+}$ para a solução do solo retira o cátion da planta e a extrusão do $\mathrm{Na}^{+}$em alguns tecidos, sobretudo o xilema, é uma estratégia que evita acúmulo do cátion no limbo foliar minimizando os efeitos deletérios sobre o metabolismo foliar, em especial sobre o processo fotossintético (Munns et al., 2002).

O presente trabalho visou avaliar os efeitos do estresse salino em genótipos tetraplóides de bananeira, por meio de avaliações biométricas e quantificação de íons inorgânicos nas plantas em fase inicial de crescimento, período de maior susceptibilidade à salinidade.

\section{Material e mÉtodos}

O experimento foi conduzido em casa de vegetação da Universidade Federal Rural de Pernambuco, avaliando-se doze genótipos tetraplóides: Calipso, FHIA-02, FHIA-03, FHIA-21, FHIA-18, PV 42-142, PA 42-44, Bucaneiro, Pacovan Ken, Tropical, Ambrosia e Preciosa (Tabela 1). Mudas provenientes de cultura de tecidos, com aproximadamente $30,0 \mathrm{~cm}$ de altura, cedidas pelo Banco Ativo de Germoplasma (BAG) da Embrapa Mandioca e Fruticultura Tropical, foram plantadas em vasos de polietileno contendo $10 \mathrm{~kg}$ de areia lavada coberta com uma camada de $3 \mathrm{~cm}$ de cascalho fino, a fim de reduzir a evaporação e favorecer o controle da salinidade no substrato. Utilizou-se irrigação por gotejamento $\left(0,6 \mathrm{~L} \mathrm{planta}^{-1} \mathrm{dia}^{-1}\right)$. A solução de rega de todos os tratamentos continha $742,86 \mathrm{mg} \mathrm{L}^{-1}$ de fertilizante solúvel (marca Kristalon ${ }^{\circledR}$ ) com a seguinte composição: $30 \mathrm{~g} \mathrm{~kg}^{-1}$ de $\mathrm{N}, 110 \mathrm{~g} \mathrm{~kg}^{-1}$ de $\mathrm{P}_{2} \mathrm{O}_{5}, 380 \mathrm{~g} \mathrm{~kg}^{-1}$ de $\mathrm{K}_{2} \mathrm{O}, 40 \mathrm{~g} \mathrm{~kg}^{-1}$ de $\mathrm{MgO}, 110 \mathrm{~g} \mathrm{~kg}^{-1}$ de S e micronutrientes. $\mathrm{O}$ cálcio e o nitrogênio foram fornecidos na forma de nitrato de cálcio (marca Barco Viking ${ }^{\circledR}$ ) na dose de $840 \mathrm{mg} \mathrm{L}^{-1}$ do produto que contém $15 \%$ de $\mathrm{Ne} 19 \%$ de Ca. A condutividade elétrica da

Tabela 1. G rupo genômico, geneal ogia e atributos dos genótipos tetraplóides avaliados

\begin{tabular}{|c|c|c|c|}
\hline Genótipos & Genoma & Genealogia (Origem) & Atributos do genótipo \\
\hline FHIA-02 & AAAA & $\begin{array}{l}\text { Híbrido de Cavendish } \\
\text { (FHIA) }\end{array}$ & $\begin{array}{l}\text { Frutos semelhantes aos de Grande Naine, resistente ao mal-do-Panamá, moderadamente } \\
\text { resistente a Sigatoka-amarela e a Sigatoka-negra. }\end{array}$ \\
\hline Calipso & AAAA & $\begin{array}{l}\text { Híbrido High Gate } \\
\text { (J amaica) }\end{array}$ & \multirow{3}{*}{$\begin{array}{l}\text { Fruto semelhante à banana d'água (Cavendish), não testado no mercado, resistente a Sigatoka- } \\
\text { negra e ao mal-do-Panamá. }\end{array}$} \\
\hline Bucanero & AAAA & $\begin{array}{l}\text { Híbrido High Gate } \\
\text { (J amaica) }\end{array}$ & \\
\hline Ambrosia & AAAA & $\begin{array}{l}\text { Híbrido High Gate } \\
\text { (J amaica) }\end{array}$ & \\
\hline FHIA-03 & AAAB & $\begin{array}{l}\text { Híbrido tipo Figo da } \\
\text { FHIA-2 }\end{array}$ & $\begin{array}{l}\text { Frutos com casca grossa, semelhantes aos da Figo, que também podem ser consumidos crus. A } \\
\text { planta é resistente a Sigatoka-negra, Sigatoka-amarela e mal-do-Panamá. }\end{array}$ \\
\hline PA42-44 & AAAB & $\begin{array}{l}\text { Híbrido de Prata Anã } \\
\text { (FHIA) }\end{array}$ & Frutos muito semelhantes aos da Prata Anã, resistente à Sigatoka-amarela. \\
\hline $\begin{array}{l}\text { Preciosa } \\
\text { (PV42-85) }\end{array}$ & AAAB & $\begin{array}{l}\text { 'Pacovan' XM53- } \\
\text { EMBRAPA }\end{array}$ & \multirow{3}{*}{$\begin{array}{l}\text { Apresentam planta alta com frutos semelhantes aos de Pacovan. Resistentes às Sigatokas } \\
\text { amarela e negra e ao mal-do-Panamá. São semelhantes entre si, diferindo em pequenos } \\
\text { caracteres agronômicos. A Preciosa e Pacovan Ken já foram recomendadas. }\end{array}$} \\
\hline $\begin{array}{l}\text { Pacovan Ken } \\
\text { (PV42-68) }\end{array}$ & AAAB & $\begin{array}{l}\text { 'Pacovan X M } 53- \\
\text { EMBRAPA }\end{array}$ & \\
\hline $\begin{array}{l}\text { Japira } \\
\text { (PV42-142) }\end{array}$ & AAAB & $\begin{array}{l}\text { 'Pacovan' X M53- } \\
\text { EMBRAPA }\end{array}$ & \\
\hline $\begin{array}{l}\text { Tropical } \\
\text { (YB42-21) }\end{array}$ & AAAB & $\begin{array}{l}\text { Yangambi XM53 } \\
\text { EMBRAPA }\end{array}$ & Híbrido tipo Maçã, tolerante ao mal-do-Panamá e resistente a Sigatoka-amarela \\
\hline FHIA-18 & AAAB & $\begin{array}{l}\text { Híbrido de Prata Anã } \\
\text { (FHIA) }\end{array}$ & $\begin{array}{l}\text { Frutos tipo prata que despencam com facilidade, resistente a Sigatoka-negra, moderadamente } \\
\text { resistente a Sigatoka-amarela e suscetível ao mal-do-Panamá,. }\end{array}$ \\
\hline FHIA-21 & AAAB & $\begin{array}{l}\text { Híbrido tipo Terra } \\
\text { (FHIA) }\end{array}$ & $\begin{array}{l}\text { Planta com fruto tipo Terra, porte médio, resistente à Sigatoka amarela, mal-do-Panamá e } \\
\text { Sigatoka-negra }\end{array}$ \\
\hline
\end{tabular}


solução nutritiva foi mantida a 1,3 e 11,4 dS m $\mathrm{d}^{-1}$, correspondendo às concentrações de 0 e $100 \mathrm{mM}$ de $\mathrm{NaCl}$, o que caracterizou dois tratamentos. A adição de $\mathrm{NaCl}$ à solução nutritiva foi realizada em duas etapas, ajustando-se as seguintes concentrações: $50 \mathrm{mM}$ no início do tratamento e $100 \mathrm{mM}$ após uma semana. Utilizou-se um fatorial 12 x 2 em um delineamento experimental inteiramente casualizado, com cinco repetições.

Avaliaram-se, após vinte e um dias de tratamento, os atributos de crescimento: diâmetro do pseudocaule, medido com o auxílio de um paquímetro; área foliar (obtida a partir do produto da largura e do comprimento das folhas pelo fator de correção 0,7$)$; altura da planta e número de folhas. Por ocasião da coleta obteve-se, separadamente, a biomassa seca do limbo foliar, do pseudocaule e das raízes com rizoma (raízes + rizoma). A biomassa seca foi determinada após secagem das partes das plantas em estufa a $65^{\circ} \mathrm{C}$, até peso constante, utilizando-se balança digital. Para análise dos elementos minerais, a biomassa seca das diferentes partes da planta foi triturada em moinho de facas e submetida a digestão nitro-perclórica. $\mathrm{O}$ extrato vegetal foi dissolvido e diluído em água deionizada, para determinação dos teores de $\mathrm{Na}^{+}, \mathrm{K}^{+}$e $\mathrm{Ca}^{2+}$. Os teores de $\mathrm{Na}^{+}$e $\mathrm{K}^{+}$foram obtidos por fotometria de chama e a determinação de $\mathrm{Ca}^{2+}$ por espectrofotometria de absorção atômica. O teor de cloreto foi determinado por titulometria do nitrato de prata (Gomes et al., 2002).

Os dados obtidos foram submetidos a análise de variância e as médias comparadas pelo teste de Tukey a $5 \%$ de probabilidade.

\section{RESULTADOS E DISCUSSÃO}

A maioria dos genótipos submetidos ao estresse salino apresentou reduções na produção de biomassa seca (Tabela 2). A redução do crescimento, destacando-se a produção da fitomassa, está relacionada tanto com a componente osmótica como com a componente iônica, ambas indissociáveis no estresse salino. A baixa disponibilidade de água resultante da redução do potencial osmótico devido à elevada concentração salina, provoca o fechamento dos estômatos e, consequentemente, reduz a asssimilação do $\mathrm{CO}_{2}$ e a taxa fotossintética (Willadino \& Camara, 2004). No que se refere à componente iônica, a homeostase exige um consumo considerável de energia para manter a compartimentalização do sódio, tanto nas células quanto nos órgãos. A associação desses fatores é determinante na redução do crescimento. Destacam-se, neste contexto, os genótipos Preciosa e FHIA18, por não apresentarem reduções significativas da matéria seca, seja no limbo ou no pseudocaule, quando submetidas a estresse salino. Nesses dois genótipos não se observaram sintomas de toxidez.

A ausência de sintomatologia foi observada também nos genótipos Pacovan Ken, Tropical, Ambrosia e FHIA-21, caracterizando uma adaptação maior ao $\mathrm{NaCl}$ neste grupo de genótipos. A maior parte desses genótipos não apresentou redução significativa na área foliar (Tabela 3). Por outro lado, dos doze genótipos estudados seis apresentaram sintomas visuais de toxidez causada pelo tratamento salino: Calipso, FHIA-02, PA 42-44, Bucaneiro, FHIA-03 e PV 42-142. Os sintomas foram caracterizados pela presença de pequenas manchas cloróticas, ao longo do limbo foliar, que evoluíram para necrose das bordas e do ápice foliar. Dos genótipos que manifestaram sintomatologia, grande parte apresentou redução significativa na área foliar (16,7 a 55,2\%), atributo de maior sensibilidade à salinidade. De maneira geral, a redução da área foliar em função do incremento dos níveis de sal, é uma resposta frequente em diversos genótipos de bananeira (Gomes et al., 2002; 2005; Carmo et al., 2003; Silva et al., 2009). A menor produtividade da bananeira sob estresse salino (Gondim et al., 2006) está relacionada, provavelmente, à redução da área foliar uma vez que este fator é limitante, no que se refere à produção de fotossintatos.

A manutenção da área foliar se relaciona, na maioria dos genótipos aclimatados, com uma redução do número de folhas, o que indica uma priorização da expansão foliar em detrimento da emissão de novas folhas.

As variáveis altura e diâmetro do pseudocaule (Tabela 3), não foram consideradas bons indicadores morfofisiológicos

Tabela 2. Biomassa seca (g) do limbo foliar, pseudocaule e raízes+ rizoma em doze genótipos de bananeira, em estádio inicial de desenvolvimento, aos 21 dias de tratamento salino ${ }^{1}$

\begin{tabular}{|c|c|c|c|c|c|c|}
\hline \multirow{4}{*}{ Genótipos } & \multicolumn{2}{|c|}{ Limbo Foliar } & \multirow{2}{*}{\multicolumn{2}{|c|}{$\begin{array}{c}\text { Pseudocaule } \\
\mathrm{NaCl}(\mathrm{mM})\end{array}$}} & \multicolumn{2}{|c|}{ Raízes+ Rizoma } \\
\hline & & & & & & \\
\hline & 0 & 100 & 0 & 100 & 0 & 100 \\
\hline & \multicolumn{6}{|c|}{ Biomassa seca (g) } \\
\hline Pacovan Ken & $17,05 \mathrm{Abc}$ & $13,22 \mathrm{Bbcd}$ & $10,45 \mathrm{Ab}$ & $8,39 \mathrm{Bd}$ & $10,27 \mathrm{aA}$ & $7,89 \mathrm{aA}$ \\
\hline Tropical & $18,64 \mathrm{Aabc}$ & $13,37 \mathrm{Bbcd}$ & $11,84 \mathrm{Ab}$ & $9,36 \mathrm{Bcd}$ & $14,19 \mathrm{aA}$ & $7,31 \mathrm{aA}$ \\
\hline Ambrosia & $19,09 \mathrm{Aabc}$ & $16,09 \mathrm{Bab}$ & $15,19 \mathrm{Aa}$ & $11,25 \mathrm{Babc}$ & $21,94 \mathrm{aA}$ & $12,15 \mathrm{aB}$ \\
\hline FHIA-21 & $19,29 \mathrm{Aab}$ & $13,31 \mathrm{Bbcd}$ & $10,21 \mathrm{Ab}$ & $9,12 \mathrm{Acd}$ & $11,01 \mathrm{aA}$ & $8,61 \mathrm{aA}$ \\
\hline FHIA-18 & 16,14 Aab & 15,44 Aabc & $12,57 \mathrm{Ab}$ & $9,45 \mathrm{Acd}$ & $14,57 \mathrm{aA}$ & $10,72 \mathrm{aA}$ \\
\hline Preciosa & $15,58 \mathrm{AC}$ & 14,97 Aabc & $10,51 \mathrm{Ab}$ & $9,85 \mathrm{Abd}$ & $11,64 \mathrm{aA}$ & $12,09 \mathrm{aA}$ \\
\hline Calipso & 19,44 Aab & $14,57 \mathrm{Babc}$ & $12,57 \mathrm{Ab}$ & $12,05 \mathrm{Aa}$ & $10,69 \mathrm{aA}$ & $11,39 \mathrm{aA}$ \\
\hline FHIA-02 & $18,86 \mathrm{Aabc}$ & $12,58 \mathrm{Bcd}$ & $12,18 \mathrm{Ab}$ & $9,57 \mathrm{Bcd}$ & $13,13 \mathrm{aA}$ & $11,18 \mathrm{aA}$ \\
\hline PA 42-44 & $20,69 \mathrm{Aa}$ & $16,87 \mathrm{Ba}$ & $12,53 \mathrm{Ab}$ & $11,90 A a b$ & $16,72 \mathrm{aA}$ & $15,74 \mathrm{aA}$ \\
\hline Bucaneiro & $14,74 \mathrm{AC}$ & $11,86 \mathrm{Bd}$ & $11,69 \mathrm{Ab}$ & $8,81 \mathrm{Bd}$ & $20,03 \mathrm{aA}$ & $10,87 \mathrm{aB}$ \\
\hline FHIA-03 & $16,67 \mathrm{Abc}$ & $12,36 \mathrm{Bd}$ & $12,15 \mathrm{Ab}$ & $9,27 \mathrm{Bcd}$ & $16,91 \mathrm{aA}$ & $11,77 \mathrm{aA}$ \\
\hline PV 42-142 & $14,64 \mathrm{Ac}$ & $12,73 \mathrm{Bcd}$ & $10,22 \mathrm{Ab}$ & $8,49 \mathrm{Bd}$ & $15,78 \mathrm{aA}$ & $13,74 \mathrm{aA}$ \\
\hline
\end{tabular}

${ }^{1}$ Médias seguidas das mesmas letras, minúsculas nas colunas e maiúsculas nas linhas (dentro de cada parte da planta), não diferem entre si pelo teste de Tukey a $5 \%$ de probabilidade 
Tabela 3. N úmero de folhas, altura $(\mathrm{cm})$, diâmetro do pseudocaule $(\mathrm{cm})$ e área foliar $\left(\mathrm{cm}^{2}\right)$ em doze genótipos de bananeira, em estádio inicial de desenvolvimento, aos 21 dias de tratamento salino ${ }^{1}$

\begin{tabular}{|c|c|c|c|c|c|c|c|c|}
\hline \multirow{3}{*}{ Genótipos } & \multicolumn{2}{|c|}{ Número de folhas } & \multicolumn{2}{|c|}{ Altura $(\mathrm{cm})$} & \multicolumn{2}{|c|}{ Diâmetro do pseudocaule } & \multicolumn{2}{|c|}{ Área foliar $\left(\mathrm{cm}^{2}\right)$} \\
\hline & \multicolumn{8}{|c|}{$\mathrm{NaCl}(\mathrm{mM})$} \\
\hline & 0 & 100 & 0 & 100 & 0 & 100 & 0 & 100 \\
\hline Tropical & 8,66 aA & $6,33 a B$ & $31,00 \mathrm{abA}$ & $21,83 \mathrm{aB}$ & $2,66 \mathrm{aA}$ & $2,04 \mathrm{aB}$ & $2101,13 \mathrm{aA}$ & $1353,00 \mathrm{aB}$ \\
\hline Ambrosia & $8,33 \mathrm{aA}$ & $7,33 \mathrm{aA}$ & $32,50 \mathrm{abA}$ & $27,00 \mathrm{aA}$ & $2,95 \mathrm{aA}$ & $2,31 \mathrm{aB}$ & $2532,73 \mathrm{aA}$ & $1848,58 a A$ \\
\hline FHIA-21 & $8,33 \mathrm{aA}$ & $7,00 \mathrm{aB}$ & $24,56 \mathrm{abA}$ & $20,66 \mathrm{aA}$ & $2,35 \mathrm{aA}$ & $2,11 \mathrm{aA}$ & $1333,51 \mathrm{aA}$ & $1496,08 \mathrm{aA}$ \\
\hline Preciosa & 9,33 aA & $7,66 \mathrm{aB}$ & $34,33 \mathrm{aA}$ & $25,16 \mathrm{aB}$ & $2,56 \mathrm{aA}$ & $2,32 \mathrm{aA}$ & $2598,61 \mathrm{aA}$ & $1930,54 \mathrm{aA}$ \\
\hline Calipso & $7,66 \mathrm{aA}$ & $7,00 \mathrm{aA}$ & $27,33 \mathrm{abA}$ & $26,33 \mathrm{aA}$ & $2,28 \mathrm{aA}$ & $2,21 \mathrm{aA}$ & $1966,65 \mathrm{aA}$ & $1638,72 \mathrm{aA}$ \\
\hline FHIA-02 & 9,33 aA & $7,66 \mathrm{aB}$ & $26,50 \mathrm{abA}$ & $17,00 \mathrm{aB}$ & $2,56 \mathrm{aA}$ & $2,06 \mathrm{aB}$ & $2253,79 \mathrm{aA}$ & $1418,80 \mathrm{aB}$ \\
\hline PA 42-44 & 9,33 aA & $8,33 \mathrm{aA}$ & $25,76 \mathrm{abA}$ & 22,61 aA & $2,26 \mathrm{aA}$ & $2,50 \mathrm{aA}$ & $2427,61 \mathrm{aA}$ & $1700,55 \mathrm{aB}$ \\
\hline Bucaneiro & $9,00 \mathrm{aA}$ & $6,33 \mathrm{aB}$ & $31,69 a b A$ & $19,00 \mathrm{aB}$ & $2,67 \mathrm{aA}$ & $2,01 a B$ & $2697,42 \mathrm{aA}$ & $1208,70 \mathrm{aB}$ \\
\hline FHIA-03 & $8,66 \mathrm{aA}$ & $7,33 \mathrm{aB}$ & $27,60 \mathrm{abA}$ & $17,33 a B$ & $2,84 \mathrm{aA}$ & $2,24 \mathrm{aB}$ & $2448,06 \mathrm{aA}$ & $1635,18 \mathrm{aB}$ \\
\hline PV 42-142 & 9,33 aA & $7,66 \mathrm{aA}$ & $21,93 \mathrm{bA}$ & 19,36 aA & $2,26 \mathrm{aA}$ & $2,14 \mathrm{aA}$ & $1812,98 \mathrm{aA}$ & 1449,36 aA \\
\hline
\end{tabular}

${ }^{1}$ Médias seguidas das mesmas letras minúsculas nas colunas e maiúsculas nas linhas, não diferem entre si pelo teste de Tukey a $5 \%$ de probabilidade

para tolerância ou sensibilidade à salinidade, uma vez que não diferenciaram os genótipos que apresentam, ou não, sintomatologia de toxidez ao $\mathrm{Na}^{+}$.

As Tabelas 4 e 5 apresentam os teores de $\mathrm{Na}^{+}, \mathrm{K}^{+}, \mathrm{Ca}^{2+}$ e $\mathrm{Cl}^{-}$ no limbo foliar, pseudocaule e raiz+rizoma das plantas tratadas com e sem $\mathrm{NaCl}$. No que se refere aos teores de sódio observou-se, em todos os genótipos, um acúmulo significativo desse íon em todas as partes da planta (Tabela 4). O grande diferencial entre os genótipos adaptados e sensíveis se refere à magnitude do incremento no teor desse íon. Os genótipos mais aclimatados apresentaram teores de sódio no limbo foliar que variaram de 2,52 a 5,80 $\mathrm{g} \mathrm{kg}^{-1}$, enquanto os mais sensíveis apresentaram variações da ordem de 3,30 a $10,53 \mathrm{~g} \mathrm{~kg}^{-1}$.

Dentre os genótipos mais sensíveis se destaca o PA 42-44 com o maior incremento no teor de sódio, tanto no limbo foliar (406\%) quanto nas raízes e rizoma (673\%). Incrementos similares nos teores de $\mathrm{Na}^{+}$do limbo foliar (254 a 638\%) foram observados em genótipos diplóides de bananeira, quando submetidos a $100 \mathrm{mM}$ de $\mathrm{NaCl}$ (Gomes et al., 2002). Em contraste com o genótipo PA 42-44 se ressalta Preciosa, com os menores incrementos de sódio, tanto no limbo foliar quanto nas raízes e rizoma. Tendo em vista a inevitável absorção de sódio pelas

Tabela 4. Teores de $\mathrm{Na}^{+}$e $\mathrm{K}^{+}$no limbo folhiar, pseudocaule e raízes+ rizoma em doze genótipos de bananeira, em estádio inicial de desenvolvimento, aos 21 dias de tratamento salino ${ }^{1}$

\begin{tabular}{|c|c|c|c|c|c|c|}
\hline \multirow{3}{*}{ Genótipos } & \multicolumn{2}{|c|}{ Limbo foliar } & \multicolumn{2}{|c|}{ Pseudocaule } & \multicolumn{2}{|c|}{ Raízes+ Rizoma } \\
\hline & \multicolumn{6}{|c|}{$\mathrm{NaCl}(\mathrm{mM})$} \\
\hline & 0 & 100 & 0 & 100 & 0 & 100 \\
\hline & \multicolumn{6}{|c|}{$\mathrm{Na}^{+}\left(\mathrm{g} \mathrm{kg}^{-1}\right)$} \\
\hline Pacovan Ken & $2,93 a B$ & $4,09 \mathrm{cdA}$ & $2,43 a B$ & $9,47 \mathrm{cA}$ & $0,65 a B$ & $10,69 \mathrm{aA}$ \\
\hline Tropical & $1,37 \mathrm{bB}$ & $4,79 \mathrm{cA}$ & $1,6 \mathrm{bcB}$ & $4,74 \mathrm{fA}$ & $1,32 \mathrm{aB}$ & $4,01 \mathrm{eA}$ \\
\hline Ambrosia & $0,69 \mathrm{bB}$ & $3,36 \mathrm{de} A$ & 1,12 bcB & $10,87 \mathrm{aA}$ & $0,40 \mathrm{aB}$ & $8,50 \mathrm{bcA}$ \\
\hline FHIA-21 & $0,30 \mathrm{bB}$ & $4,02 \mathrm{cdA}$ & $0,51 \mathrm{cB}$ & $9,47 \mathrm{CA}$ & $1,20 \mathrm{aB}$ & $9,44 a b c A$ \\
\hline FHIA-18 & $3,33 \mathrm{aB}$ & $5,80 \mathrm{bA}$ & $0,82 \mathrm{cB}$ & $6,66 \mathrm{eA}$ & $1,33 a B$ & $10,62 \mathrm{abA}$ \\
\hline Preciosa & $0,76 \mathrm{bB}$ & $2,52 \mathrm{eA}$ & $0,44 c B$ & $6,68 \mathrm{eA}$ & $1,16 \mathrm{aB}$ & 4,82 eA \\
\hline Calipso & $0,23 \mathrm{bB}$ & $4,90 \mathrm{cA}$ & $0,70 \mathrm{cB}$ & $10,73 a A$ & $1,00 \mathrm{aB}$ & $7,56 \mathrm{cdA}$ \\
\hline FHIA-02 & $0,81 \mathrm{bB}$ & $3,58 \mathrm{cde} A$ & $0,41 \mathrm{cB}$ & $5,38 \mathrm{fA}$ & $0,97 \mathrm{aB}$ & $5,80 \mathrm{deA}$ \\
\hline PA 42-44 & $0,15 \mathrm{bB}$ & $10,53 \mathrm{aA}$ & $0,44 \mathrm{cB}$ & $9,63 \mathrm{bcA}$ & $0,58 \mathrm{aB}$ & $11,08 \mathrm{aA}$ \\
\hline Bucaneiro & $0,19 \mathrm{bB}$ & $4,60 \mathrm{cdA}$ & $1,83 a b B$ & $3,80 \mathrm{gA}$ & $1,03 \mathrm{aB}$ & $9,40 a b c A$ \\
\hline FHIA-03 & $0,46 \mathrm{bB}$ & 3,30 deA & $0,40 c B$ & $10,43 a b A$ & $0,27 a B$ & 4,34 eA \\
\hline \multirow[t]{2}{*}{ PV $42-142$} & $0,16 \mathrm{bB}$ & $6,50 \mathrm{bA}$ & $1,10 \mathrm{bcB}$ & $8,52 \mathrm{dA}$ & $1,44 a \mathrm{a}$ & $9,70 \mathrm{abcA}$ \\
\hline & \multicolumn{6}{|c|}{$\mathrm{K}^{+}\left(\mathrm{g} \mathrm{kg}^{-1}\right)$} \\
\hline Pacovan Ken & $69,33 \mathrm{aA}$ & $40,72 \mathrm{deB}$ & $82,05 b c B$ & $152,20 \mathrm{abA}$ & $65,12 \mathrm{abB}$ & $103,76 \mathrm{bA}$ \\
\hline Tropical & 57,86 abcB & $79,00 \mathrm{abA}$ & $86,66 \mathrm{bcB}$ & $123,66 \mathrm{abcdA}$ & 38,73 bcdB & $66,76 \mathrm{cA}$ \\
\hline Ambrosia & $52,39 \mathrm{abcdB}$ & 64,09 bcA & 79,56 bcB & 120,30 bcdA & $17,36 \mathrm{~dB}$ & $49,23 \mathrm{CA}$ \\
\hline FHIA-21 & $64,26 \mathrm{abA}$ & $57,50 \mathrm{cdA}$ & $112,13 \mathrm{abB}$ & 139,06 abcA & $68,09 a B$ & $136,13 \mathrm{aA}$ \\
\hline FHIA-18 & $25,61 \mathrm{eA}$ & 34,28 eA & $106,31 \mathrm{abA}$ & $124,29 \mathrm{abcdA}$ & $44,43 \mathrm{abcdB}$ & $68,55 \mathrm{cA}$ \\
\hline Preciosa & $26,13 \mathrm{eB}$ & 65,66 bcA & 95,65 bcA & $132,15 \mathrm{abcdA}$ & 57,86 abA & $46,31 \mathrm{cA}$ \\
\hline Calipso & 67,96 aA & 75,20 abcA & $132,19 a \mathrm{~A}$ & $115,43 \mathrm{cdA}$ & 49,74 abcA & $63,06 \mathrm{cA}$ \\
\hline FHIA-02 & $48,53 \mathrm{bcdB}$ & 91,66 aA & $106,96 \mathrm{abB}$ & $149,90 \mathrm{abA}$ & $59,53 \mathrm{abA}$ & $44,36 \mathrm{cdA}$ \\
\hline PA 42-44 & 47,89 bcdB & $72,63 \mathrm{bcA}$ & $69,36 \mathrm{cdB}$ & $148,50 \mathrm{abA}$ & $25,53 \mathrm{cdB}$ & $65,66 \mathrm{cA}$ \\
\hline Bucaneiro & $42,13 \mathrm{cdeB}$ & 63,86 bcA & $65,66 \mathrm{cdB}$ & $115,36 \mathrm{cdA}$ & $25,89 \mathrm{cdA}$ & $17,57 \mathrm{dA}$ \\
\hline FHIA-03 & $36,23 \mathrm{deB}$ & 70,89 bcA & $39,16 \mathrm{~dB}$ & $154,20 \mathrm{aA}$ & 39,36 bcdA & $46,90 \mathrm{cA}$ \\
\hline PV 42-142 & $47,89 \mathrm{bcdB}$ & 73,23 bcA & $68,66 \mathrm{cdB}$ & $100,62 \mathrm{dA}$ & $24,89 \mathrm{cdB}$ & $44,23 \mathrm{cdA}$ \\
\hline
\end{tabular}

${ }^{1}$ Médias seguidas das mesmas letras minúsculas nas colunas e maiúsculas nas linhas, não diferem entre si pelo teste de Tukey a $5 \%$ de probabilidade 
Tabela 5. Teores de $\mathrm{Cl}^{-}$e $\mathrm{Ca}^{+2}$ no limbo folhiar, pseudocaule e raízes+ rizoma em doze genótipos de bananeira, em estádio inicial de desenvolvimento, aos 21 dias de tratamento salino ${ }^{1}$

\begin{tabular}{|c|c|c|c|c|c|c|}
\hline \multirow{3}{*}{ Genótipos } & \multicolumn{2}{|c|}{ Limbo foliar } & \multirow{2}{*}{\multicolumn{2}{|c|}{ Pseudocaule }} & \multicolumn{2}{|c|}{ Raízes+ Rizoma } \\
\hline & & & & & & \\
\hline & 0 & 100 & 0 & 100 & 0 & 100 \\
\hline & \multicolumn{6}{|c|}{$\mathrm{Cl}^{-}\left(\mathrm{g} \mathrm{kg}^{-1}\right)$} \\
\hline Pacovan Ken & $3,86 \mathrm{aB}$ & $25,50 \mathrm{deA}$ & $3,86 \mathrm{aB}$ & $45,53 \mathrm{cA}$ & $6,70 \mathrm{aA}$ & $45,96 \mathrm{abA}$ \\
\hline Tropical & $3,76 \mathrm{aB}$ & $21,7 \mathrm{eA}$ & $3,70 \mathrm{aB}$ & $29,79 \mathrm{fA}$ & $3,60 \mathrm{aA}$ & $32,16 \mathrm{bA}$ \\
\hline Ambrosia & $3,96 \mathrm{aB}$ & $25,63 \mathrm{deA}$ & $3,63 a B$ & $42,03 \mathrm{cdeA}$ & $3,23 \mathrm{aB}$ & $47,83 \mathrm{aA}$ \\
\hline FHIA-21 & $4,46 \mathrm{aB}$ & $29,00 \mathrm{cdA}$ & $3,73 \mathrm{aB}$ & $60,20 \mathrm{aA}$ & $4,73 \mathrm{aA}$ & $43,50 \mathrm{abA}$ \\
\hline FHIA-18 & $5,43 \mathrm{aB}$ & $34,00 \mathrm{abA}$ & $3,57 \mathrm{aB}$ & 48,24 bcA & $4,54 \mathrm{aA}$ & $40,98 \mathrm{abA}$ \\
\hline Preciosa & $6,50 \mathrm{aB}$ & 30,18 bcA & $3,20 \mathrm{aB}$ & 35,55 efA & $8,56 \mathrm{aA}$ & $43,04 a b A$ \\
\hline Calipso & $4,69 \mathrm{aB}$ & $33,39 \mathrm{abA}$ & $3,74 \mathrm{aB}$ & $52,39 \mathrm{bA}$ & $4,26 \mathrm{aA}$ & $42,86 \mathrm{abA}$ \\
\hline FHIA-02 & $3,60 \mathrm{aB}$ & 25,33 deA & $6,70 \mathrm{aB}$ & 36,63 eA & $3,26 \mathrm{aA}$ & $38,96 \mathrm{abA}$ \\
\hline PA 42-44 & $3,60 \mathrm{aB}$ & $26,43 \mathrm{cdA}$ & $4,46 \mathrm{aB}$ & $43,50 \mathrm{cdA}$ & $4,13 \mathrm{aA}$ & $22,53 \mathrm{bA}$ \\
\hline Bucaneiro & $3,53 \mathrm{aB}$ & $37,12 \mathrm{aA}$ & $3,66 \mathrm{aB}$ & 36,28 efA & $3,50 \mathrm{aA}$ & $36,53 \mathrm{abA}$ \\
\hline FHIA-03 & $3,66 \mathrm{aB}$ & $36,50 \mathrm{aA}$ & $3,60 \mathrm{aB}$ & 36,96 deA & $3,66 \mathrm{aA}$ & $35,89 \mathrm{abA}$ \\
\hline \multirow[t]{2}{*}{ PV 42-142 } & $3,60 \mathrm{aB}$ & $36,80 \mathrm{aA}$ & $3,56 \mathrm{aB}$ & 36,22 efA & $3,66 \mathrm{aA}$ & $36,89 \mathrm{abA}$ \\
\hline & \multicolumn{6}{|c|}{$\mathrm{Ca}^{+2}\left(\mathrm{~g} \mathrm{~kg}^{-1}\right)$} \\
\hline Pacovan Ken & $8,66 \mathrm{abA}$ & $8,43 \mathrm{bA}$ & $5,90 \mathrm{bcA}$ & $5,40 \mathrm{bcA}$ & 7,73 abcA & $3,26 \mathrm{bcdB}$ \\
\hline Tropical & $5,23 \mathrm{bcB}$ & $8,33 \mathrm{bA}$ & $3,73 \mathrm{CB}$ & $8,50 \mathrm{abA}$ & 5,60 bcdeA & 4,70 bcdA \\
\hline Ambrosia & $6,44 a b c A$ & $6,60 \mathrm{bcA}$ & $5,00 \mathrm{cA}$ & 4,46 bcA & $2,93 \mathrm{eA}$ & $2,43 \mathrm{dA}$ \\
\hline FHIA-21 & $5,40 \mathrm{bcB}$ & $13,36 \mathrm{aA}$ & $3,06 \mathrm{CA}$ & $3,16 \mathrm{CA}$ & $3,63 \mathrm{deB}$ & $5,63 \mathrm{bA}$ \\
\hline FHIA-18 & $8,36 \mathrm{abA}$ & $4,50 \mathrm{cdB}$ & 6,53 abcA & $5,56 \mathrm{bcA}$ & $6,43 \mathrm{bcdA}$ & $3,53 \mathrm{bcdB}$ \\
\hline Preciosa & 6,43 abcA & $3,47 \mathrm{cdB}$ & $5,40 \mathrm{CA}$ & $3,84 \mathrm{CA}$ & $4,10 \mathrm{deA}$ & $3,50 \mathrm{bcdA}$ \\
\hline Calipso & $5,91 \mathrm{abcB}$ & $9,63 \mathrm{bA}$ & $10,06 \mathrm{abA}$ & $11,16 \mathrm{aA}$ & $10,20 \mathrm{~A}$ & $8,50 \mathrm{aB}$ \\
\hline FHIA-02 & $4,80 \mathrm{cA}$ & $2,93 \mathrm{dA}$ & $9,73 \mathrm{abA}$ & $10,86 \mathrm{aA}$ & $8,19 a b A$ & $5,40 \mathrm{bcB}$ \\
\hline PA 42-44 & 5,52 abcA & $4,73 \mathrm{cdA}$ & $5,00 \mathrm{cB}$ & $10,63 \mathrm{aA}$ & $5,38 \mathrm{cdeA}$ & $2,79 \mathrm{cdB}$ \\
\hline Bucaneiro & $8,90 \mathrm{aA}$ & $9,70 \mathrm{bA}$ & $3,63 \mathrm{CA}$ & 5,66 bcA & $3,50 \mathrm{eB}$ & 8,73 aA \\
\hline FHIA-03 & $3,23 \mathrm{cA}$ & $4,66 \mathrm{cdA}$ & $10,36 \mathrm{aA}$ & $7,13 a b c B$ & $10,13 \mathrm{aA}$ & $8,63 \mathrm{aA}$ \\
\hline PV $42-142$ & 5,76 abcA & $4,00 \mathrm{cdA}$ & 6,19 abcA & $8,43 \mathrm{abA}$ & $4,00 \mathrm{de} A$ & $4,53 b c d A$ \\
\hline
\end{tabular}

plantas submetidas a elevadas concentrações de $\mathrm{NaCl}$, a capacidade de limitar o incremento de sódio no tecido vegetal evidencia maior eficiência no processo de extrusão deste cátion. A extrusão de $\mathrm{Na}^{+}$ocorre através do antiporte $\mathrm{Na}^{+} / \mathrm{H}^{+}$, que é um transporte ativo secundário que utiliza o gradiente eletroquímico estabelecido por $\mathrm{H}^{+}$-ATPase de membrana (bombas de transporte ativo primário). A maior atividade dessas bombas já foi constatada em genótipos de bananeira com maior tolerância à salinidade (Suassuna, 2007). As bombas atuam não só em células radiculares, promovendo a extrusão do cátion para a solução do solo, mas também em células do xilema, evitando a translocação do mesmo para a parte aérea minimizando, assim, os efeitos deletérios sobre o metabolismo foliar, sobretudo o processo fotossintético (Munns et al., 2002). A bananeira Preciosa, enquadrada no grupo dos genótipos aclimatados, apresentou baixos teores de $\mathrm{Na}^{+}$no limbo foliar e na raiz e rizoma, demonstrando a eficiência do mecanismo de extrusão e de translocação desse cátion. Os genótipos Ambrósia, Pacovan Ken e FHIA-21, também adaptadas, apresentaram baixo teor de $\mathrm{Na}^{+}$no limbo foliar e elevado teor nas raízes e rizomas refletindo o controle da translocação do $\mathrm{Na}^{+}$, mediante o acúmulo do mesmo nas raízes e rizoma. Os elevados teores de sódio detectados no genótipo PA 42-44, genótipo mais sensível, sugerem uma eficiência menor na extrusão do cátion paralelamente à ausência de habilidade em evitar sua translocação para o limbo foliar.

A maioria dos genótipos aclimatados (Tropical, Pakovan Ken, Ambrosia e FHIA-21), quando submetidos ao estresse salino, apresentou aumento nos teores de cálcio no limbo foliar (Tabela 5). A elevação dos teores deste cátion favoreceu a compartimentalização iônica a nível celular, evitando a fitotoxidez nas células do limbo foliar. Os genótipos sensíveis, por sua vez, quando submetidos ao estresse salino não sofreram alterações significativas no teor deste cátion aumentando, portanto, a relação $\mathrm{Na}^{+} / \mathrm{Ca}^{2+}$. Elevadas concentrações de $\mathrm{Na}^{+}$geralmente deslocam o $\mathrm{Ca}^{2+}$ nas membranas vegetais provocando distúrbios na absorção e compartimentalização iônica (Munns et al., 2002).

Os teores de cloreto aumentaram nas plantas submetidas a salinidade; entretanto, embora bastante elevados, não apresentaram, em termos quantitativos, grandes variações entre os genótipos (Tabela. 5). Vale a pena destacar, porém, que os maiores teores deste ânion no limbo foliar (36,50; 36,80 e $37,12 \mathrm{~g} \mathrm{~kg}^{-1}$ ) foram observados em três dos seis genótipos mais sensíveis a salinidade (FHIA-03, PV 42-22 e Bucaneiro, respectivamente).

De maneira geral, ocorreu incremento ou manutenção nos teores de $\mathrm{K}^{+}$quando as plantas foram submetidas a salinidade (Tabela 4). Resultados obtidos por Gomes et al. (2004) evidenciaram a capacidade de vários genótipos de bananeira de manterem o nível de $\mathrm{K}^{+}$, mesmo quando submetidos a 100 $\mathrm{mM}$ de $\mathrm{NaCl}$. A manutenção de níveis adequados de $\mathrm{K}^{+}$é essencial para a sobrevivência de plantas em ambientes salinos, por contribuir na manutenção do baixo potencial osmótico das células vegetais que é um pré-requisito para garantir a pressão de turgor que, por sua vez, determina o transporte de soluto 
Tabela 6. Relação $\mathrm{Na}^{+} / \mathrm{K}^{+}$no limbo folhiar, pseudocaule e raízes+ rizoma em genótipos de bananeira, em estádio inicial de desenvolvimento, aos 21 dias de tratamento salino ${ }^{1}$

\begin{tabular}{|c|c|c|c|c|c|c|}
\hline \multirow{4}{*}{ Genótipos } & \multicolumn{2}{|c|}{ Limbo foliar } & \multirow{2}{*}{\multicolumn{2}{|c|}{$\begin{array}{c}\text { Pseudocaule } \\
\mathrm{NaCl} \text { (mM) }\end{array}$}} & \multicolumn{2}{|c|}{ Raízes+ Rizoma } \\
\hline & & & & & & \\
\hline & 0 & 100 & 0 & 100 & 0 & 100 \\
\hline & \multicolumn{6}{|c|}{$\mathrm{Na}^{+} / \mathrm{K}^{+}$} \\
\hline Pacovan Ken & $0,04 \mathrm{bB}$ & $0,09 \mathrm{cdA}$ & $0,03 a A$ & $0,04 \mathrm{deA}$ & $0,01 \mathrm{aA}$ & $0,10 \mathrm{bA}$ \\
\hline Tropical & $0,02 \mathrm{bB}$ & 0,05 ef $A$ & $0,01 \mathrm{aB}$ & $0,03 \mathrm{eA}$ & $0,01 \mathrm{aA}$ & $0,06 \mathrm{bA}$ \\
\hline Ambrosia & $0,01 \mathrm{bB}$ & $0,05 \mathrm{fA}$ & $0,01 \mathrm{aB}$ & $0,11 \mathrm{aA}$ & $0,01 \mathrm{aA}$ & $0,18 \mathrm{abA}$ \\
\hline FHIA-21 & $0,01 \mathrm{bB}$ & 0,12 bcA & $0,01 \mathrm{aB}$ & 0,07 bcA & $0,01 \mathrm{aA}$ & $0,06 \mathrm{bA}$ \\
\hline FHIA-18 & $0,13 \mathrm{aB}$ & 0,19 aA & $0,01 \mathrm{aB}$ & $0,06 \mathrm{cdA}$ & $0,02 \mathrm{aA}$ & $0,16 a b A$ \\
\hline Preciosa & $0,02 \mathrm{bA}$ & $0,04 \mathrm{fA}$ & $0,01 \mathrm{aB}$ & $0,05 \mathrm{cde} A$ & $0,02 \mathrm{aB}$ & $0,06 \mathrm{bA}$ \\
\hline Calipso & $0,01 \mathrm{bB}$ & $0,06 \operatorname{def} A$ & $0,01 \mathrm{aB}$ & $0,09 \mathrm{abA}$ & $0,01 \mathrm{aA}$ & $0,10 \mathrm{bA}$ \\
\hline FHIA-02 & $0,01 \mathrm{bA}$ & $0,03 \mathrm{fA}$ & $0,01 \mathrm{aB}$ & $0,03 \mathrm{eA}$ & $0,01 \mathrm{aA}$ & $0,14 a b A$ \\
\hline PA 42-44 & $0,01 \mathrm{bB}$ & $0,14 \mathrm{bA}$ & $0,01 \mathrm{aB}$ & $0,06 \mathrm{cdA}$ & $0,01 \mathrm{aA}$ & $0,18 \mathrm{abA}$ \\
\hline Bucaneiro & $0,01 \mathrm{bB}$ & 0,07 def $A$ & $0,03 a A$ & $0,03 \mathrm{eA}$ & $0,03 \mathrm{aA}$ & $0,53 \mathrm{aA}$ \\
\hline FHIA-03 & $0,01 \mathrm{bB}$ & $0,04 \mathrm{fA}$ & $0,01 \mathrm{aB}$ & $0,06 \mathrm{cdA}$ & $0,01 \mathrm{aA}$ & $0,09 \mathrm{bA}$ \\
\hline PV 42-142 & $0,01 \mathrm{bB}$ & $0,08 \mathrm{de} A$ & $0,01 \mathrm{aB}$ & $0,08 \mathrm{bA}$ & $0,01 \mathrm{aA}$ & $0,21 a b A$ \\
\hline
\end{tabular}

${ }_{1}^{1}$ Médias seguidas das mesmas letras, minúsculas nas colunas e maiúsculas nas linhas, não diferem entre si pelo teste de Tukey a $5 \%$ de probabilidade

via xilema e o balanço de água na planta (Willadino \& Camara, 2004).

A Tabela 6 apresenta a relação $\mathrm{Na}^{+} / \mathrm{K}^{+}$nas diferentes partes das plantas. Os incrementos foram mais expressivos na parte aérea, na maioria dos genótipos. Esses incrementos se devem, em parte, a maior absorção de $\mathrm{Na}^{+}$, a diminuição na absorção do $\mathrm{K}^{+}$ou a ambas. A reduzida absorção de $\mathrm{Na}^{+}$, associada a baixa relação $\mathrm{Na}^{+} / \mathrm{K}^{+}$, tem sido utilizada como importante parâmetro na seleção de genótipos de trigo tolerantes ao estresse salino (Munns et al., 2002).

A bananeira Preciosa foi o único genótipo que se enquadrou simultaneamente no grupo de menores valores da relação $\mathrm{Na}^{+} /$ $\mathrm{K}^{+}$no limbo e nas raízes e rizoma $(0,06)$. Isto se deveu ao maior incremento de $\mathrm{K}^{+}$(2,5 vezes) no limbo foliar associado ao reduzido teor de $\mathrm{Na}^{+}$, que resultou na relação $\mathrm{Na}^{+} / \mathrm{K}^{+}$de 0,04, o menor valor apresentado pelos genótipos estudados (Tabela 6). A menor relação de $\mathrm{Na}^{+} / \mathrm{K}^{+}$no limbo está correlacionada com a ausência de redução da biomassa seca da parte aérea observada nesse genótipo.

\section{CONClusÕES}

1. O genótipo Preciosa evidencia a presença de estratégias de extrusão de $\mathrm{Na}^{+}$para a solução do solo e remoção de $\mathrm{Na}^{+}$do xilema; a presença dessas estratégias resulta na manutenção dos menores teores de $\mathrm{Na}^{+}$no tecido, ausência de sintomatologia de toxicidade e manutenção da produção da biomassa seca.

2. Em contraste com a Preciosa, o genótipo PA 42-44 apresenta sintomas de toxicidade a salinidade, redução na produção da biomassa seca, elevados teores de $\mathrm{Na}^{+}$e translocação deste para o limbo foliar, caracterizando-o como o genótipo de maior susceptibilidade ao estresse salino.

\section{AgradeCimentos}

Ao Conselho Nacional de Desenvolvimento Científico e Tecnológico $(\mathrm{CNPq})$, pela concessão da bolsa DCR para a segunda autora, e de produtividade em pesquisa ao primeiro e ao quinto autor.

\section{LITERATURA CITADA}

Carmo, G. A.; Medeiros, J. F. de; Tavares, J. C.; Gheyi, H. R.; Souza, A. M.; Palácio, E. A. Q. Crescimento de bananeiras sob diferentes níveis de salinidade da água de irrigação. Revista Brasileira de Fruticultura, v.25, n.3, p.513-518, 2003.

Flowers, T. J. Improving crop salt tolerance. Journal of Experimental Botany, v.55, p.307-319, 2004.

Gomes, E. W. F.; Willadino, L.; Martins, L. S. S.; Camara, T. R.; Silva, S. O. Banana genotypes under salt stress: Tolerance and sensitivity. Infomusa, v.11, n.2, p.13-18, 2002.

Gomes, E. W. F.; Willadino, L.; Martins, L. S. S.; Silva, S. O.; Camara, T. R. Variabilidade genética de genótipos de bananeira (Musa spp) submetidos ao estresse salino. Revista Brasileira de Engenharia Agrícola e Ambiental, v.9, n.2, p.171-177, 2005.

Gomes, E. W. F.; Willadino, L.; Martins, L. S. S.; Silva, S. O.; Camara, T. R.; Meunier, I. M. J. Diplóides de bananeira submetidos ao estresse salino. Pesquisa Agropecuária Brasileira, v.39, p.525-531, 2004.

Gondim, A. R. de O.; Medeiros, J. F. de; Carmo, G. A. do; Pereira, F. H. F.; Gheyi, H. R.; Tavares, J. C. Produtividade de banana submetida a diferentes níveis de salinidade da água de irrigação: Segundo ciclo. Revista Brasileira de Engenharia Agrícola e Ambiental, v.10, n.1, p.38-42, 2006.

Munns, R.; Husain, S.; Rivelli, A. R.; Richard, A. J.; Condon, A. G.; Megan, P. L.; Evans, S. L.; Schachtman, D. P.; Hare, R. A. Avenues for increasing salt tolerance of crops, and the role of physiologically based selection traits. Plant and Soil, v.247, p. $93-105,2002$.

Shi, H.; Quintero, F. J.; Pardo, J. M.; Zhu, J. K. The putative plasma membrane $\mathrm{Na}^{+} / \mathrm{H}^{+}$antiporter SOS1 controls longdistance $\mathrm{Na}^{+}$transport in plantas. The Plant Cell, v.14, p.465477, 2002. 
Silva, R. L. O.; Martins, L. S. S.; Gomes, E. W. F.; Ferraz, G. M. G.; Silva, S. O.; Willadino, L. Avaliação de diplóides de bananeira (Musa spp.) quanto à tolerância a salinidade. Revista Brasileira de Fruticultura, v.31, n.4, p.1084-1091, 2009.

Suassuna, F. Modulação das bombas de prótons pela salinidade em genótipos de bananeira: relação com o mecanismo de tolerância. Recife: UFRPE, 2004. 79p. Dissertação Mestrado
UNCTAD info comm. Market information in the commodities area. Agricultural products: Banana. http//www.unctad.org/ infocomm/anglais/banana/market.htm_04 Jun. 2009.

Willadino, L.; Camara, T. R. Origen y naturaleza de los ambientes salinos. In: Reigosa, M. J.; Pedrol, N.; Sánchez, A. (ed.). La ecofisiología vegetal - Una ciencia de síntesis. Madrid: Thompson, 2004. p.303-330. 